\title{
Concurrent ibrutinib plus venetoclax in relapsed/refractory mantle cell lymphoma: the safety run-in of the phase 3 SYMPATICO study
}

\author{
Michael Wang ${ }^{1 *} \mathbb{D}$, Radhakrishnan Ramchandren², Robert Chen ${ }^{3}$, Lionel Karlin ${ }^{4}$, Geoffrey Chong ${ }^{5}$, \\ Wojciech Jurczak ${ }^{6}$, Ka Lung Wu ${ }^{7}$, Mark Bishton ${ }^{8}$, Graham P. Collins ${ }^{9}$, Paul Eliadis ${ }^{10}$, Frédéric Peyrade ${ }^{11}$, \\ Yihua Lee ${ }^{12}$, Karl Eckert $^{12}$, Jutta K. Neuenburg ${ }^{12}$ and Constantine S. Tam ${ }^{13}$
}

\begin{abstract}
Ibrutinib plus venetoclax, given with an ibrutinib lead-in, has shown encouraging clinical activity in early phase studies in mantle cell lymphoma (MCL). The ongoing phase 3 SYMPATICO study evaluates the safety and efficacy of concurrently administered, once-daily, all-oral ibrutinib plus venetoclax in patients with relapsed/refractory MCL. A safety run-in (SRI) cohort was conducted to inform whether an ibrutinib lead-in should be implemented for the randomized portion. Patients received concurrent ibrutinib $560 \mathrm{mg}$ continuously plus venetoclax in a 5-week ramp-up to venetoclax $400 \mathrm{mg}$ for up to 2 years. The primary endpoint was occurrence of tumor lysis syndrome (TLS) and doselimiting toxicities (DLTs). The SRI cohort enrolled 21 patients; six and 15 were in low- or increased-risk categories for TLS, respectively. During the 5-week venetoclax ramp-up, three patients had DLTs, and one patient at increased risk for TLS had a laboratory TLS; no additional TLS events occurred during follow-up. With a median follow-up of 31 months, the overall response rate was $81 \%(17 / 21) ; 62 \%(13 / 21)$ of patients had a complete response. SRI data informed that the randomized portion should proceed with concurrent ibrutinib plus venetoclax, with no ibrutinib lead-in. Ibrutinib plus venetoclax demonstrated promising efficacy; no new safety signals were observed.
\end{abstract}

Trial registration: ClinicalTrials.gov, NCT03112174. Registered 13 April 2017, https://clinicaltrials.gov/ct2/show/ NCT03112174.

Keywords: Hematological cancers/lymphomas, Small molecule agents/kinase inhibitors, Ibrutinib, Venetoclax, Safety

\section{Introduction}

Targeted therapies, such as ibrutinib, have been transformative for patients with relapsed/refractory mantle cell lymphoma $(\mathrm{MCL})$ and have partially replaced traditional chemotherapy and chemoimmunotherapy regimens. However, due to MCL's aggressive disease

\footnotetext{
*Correspondence: mwang@mdanderson.org

${ }^{1}$ Department of Lymphoma and Myeloma, University of Texas MD

Anderson Cancer Center, 1515 Holcombe Blvd \#368, Houston, TX 77030, USA

Full list of author information is available at the end of the article
}

course, effective therapies that prolong progressionfree survival (PFS) and overall survival (OS) are needed. Ibrutinib, a once-daily Bruton's tyrosine kinase (BTK) inhibitor, received accelerated approval in the USA for patients with MCL who received $\geq 1$ prior therapy [1, $2]$. In patients with relapsed/refractory MCL, singleagent ibrutinib induced complete response (CR) rates of $19-28 \%$ and overall response rates (ORRs) of $70-77 \%$ [35], and demonstrated significant improvement in PFS in a phase 3 randomized study versus temsirolimus [4]. With 7.5 years of follow-up, single-agent ibrutinib demonstrated sustained efficacy and no unexpected toxicity for 
patients with relapsed/refractory MCL [5]. Venetoclax is an oral BCL-2 inhibitor approved in the USA for the treatment of patients with chronic lymphocytic leukemia and previously untreated acute myeloid leukemia [6]. In a phase 1 study of patients with relapsed/refractory MCL, venetoclax yielded a $75 \%$ ORR and a $21 \%$ CR rate [7]. Ibrutinib and venetoclax have distinct and complementary modes of action and have demonstrated synergistic antitumor activity in preclinical models of MCL, wherein the combination led to increased apoptosis (23\%) compared to each agent alone (ibrutinib, 3.8\%; venetoclax, $3.0 \%)[8]$.

As venetoclax is highly effective at inducing apoptosis, treatment is associated with an increased risk of tumor lysis syndrome (TLS) $[9,10]$, with label-recommended in-hospital monitoring for TLS development after venetoclax initiation [6]. One strategy to mitigate the risk of developing TLS post-venetoclax initiation is to employ a lead-in with a tumor-debulking agent, such as an antiCD20 antibody or single-agent ibrutinib [11, 12]. However, given the aggressive nature of $\mathrm{MCL}$, concurrent initiation of ibrutinib and venetoclax may alleviate a potential risk for disease progression (PD) during lead-in.

The phase 3 SYMPATICO study evaluates the oncedaily oral combination of ibrutinib plus venetoclax for the treatment of patients with relapsed/refractory MCL. Here, a safety run-in (SRI) was conducted to determine whether concurrent administration of ibrutinib plus venetoclax without lead-in was tolerable. Results from the SRI would inform dosing in the randomized portion of the study.

\section{Patients and methods}

SYMPATICO (PCYC-1143-CA, NCT03112174) is a phase 3 multinational study comprising an open-label SRI cohort and a double-blind randomized period, both conducted in relapsed/refractory patients. An open-label arm in previously untreated patients is currently enrolling (Additional file 1: Figure S1).

In the SRI cohort, enrolled patients (aged $\geq 18$ years) had pathologically confirmed MCL and $\geq 1$ site of disease $\geq 2.0 \mathrm{~cm} ; 1-5$ prior therapies for $\mathrm{MCL}$, including $\geq 1$ prior rituximab/anti-CD20-containing regimen; and Eastern Cooperative Oncology Group performance status of $0-2$. Patients who had received prior BTK or BCL-2 inhibitors were excluded.

Initiating on day 1 , patients received concurrent oral ibrutinib $560 \mathrm{mg}$ once daily and venetoclax starting from $20 \mathrm{mg}$ once daily and ramped up over 5 weeks to a target dose of $400 \mathrm{mg}$ (Additional file 1: Figure S1) [6]. Patients received ibrutinib plus venetoclax concurrently for 2 years followed by once-daily single-agent ibrutinib until PD, unacceptable toxicity, or withdrawal of consent. The study was conducted according to the principles of the Declaration of Helsinki and the Good Clinical Practice guidelines from the International Conference on Harmonization. The protocol was approved by institutional review boards or independent ethics committees at participating institutions, and all patients provided written informed consent.

The primary endpoint of the SRI cohort was the occurrence of TLS and dose-limiting toxicity (DLT) events. A study algorithm was developed to determine the threshold for TLS events and DLTs required to proceed with or without an ibrutinib lead-in (Additional file 1: Figure S2). TLS-risk categories were based on tumor burden and adapted from the US prescribing information for venetoclax [6]. Patients with high tumor burden $(\geq 1$ lesion $>10 \mathrm{~cm}$ or $\geq 1$ lesion $>5 \mathrm{~cm}$ with circulating lymphocytes $>25,000$ cells $/ \mathrm{mm}^{3}$ ) and/or creatinine clearance $<60 \mathrm{~mL} / \mathrm{min}$ at baseline were considered at increased risk for TLS and received TLS prophylaxis and monitoring. Patients not meeting these criteria were considered at low risk for TLS.

Laboratory TLS was assessed per Howard criteria [13]. Clinical TLS was assessed per Howard criteria [13] with modifications (i.e., a diagnosis of clinical TLS required increases in serum creatinine $>1.0 \mathrm{mg} / \mathrm{dL}$ from pretreatment baseline); see Additional file 1: Methods for details. DLT was defined as any grade $\geq 3$ non-TLS adverse event $(\mathrm{AE})$ at least possibly related to ibrutinib and/or venetoclax occurring during the 5-week ramp-up, which ended on day 7 of venetoclax $400 \mathrm{mg}$; see Additional file 1: Methods for details.

Secondary endpoints included ORR (i.e., CR, as assessed by positron emission tomography, and partial response per the 2014 Lugano criteria) [14], PFS, duration of response, and safety. Minimal residual disease (MRD) was assessed in bone marrow and peripheral blood by flow cytometry with a detection limit of $5 \times 10^{-4}$.

Requests for access to individual participant data from clinical studies conducted by Pharmacyclics LLC, an AbbVie Company, can be submitted through Yale Open Data Access Project site (http://yoda.yale.edu).

\section{Results}

The SRI enrolled 21 patients (Table 1). Six patients (29\%) were considered at low risk for TLS and 15 patients (71\%) at increased risk for TLS. All patients had confirmed PD and $\geq 1$ lesion $>2 \mathrm{~cm}$ at baseline; 11 patients (52\%) had baseline detectable MRD in peripheral blood or bone marrow. For patients with available TP53 mutation data, 5/13 (38\%) had mutated TP53. Median followup was 31 months (range, 1.5+ to 40.2) (Additional file 1: Table S1). 
Table 1 Baseline demographic and disease characteristics

\begin{tabular}{|c|c|c|c|}
\hline & $\begin{array}{l}\text { Patients at Low Risk for TLS } \\
n=6\end{array}$ & $\begin{array}{l}\text { Patients at Increased Risk for } \\
\text { TLS } \\
n=15^{\text {a }}\end{array}$ & $\begin{array}{l}\text { All Patients } \\
N=21\end{array}$ \\
\hline Median age, years (range) & $62(54-67)$ & $70(53-84)$ & $68(53-84)$ \\
\hline \multicolumn{4}{|l|}{ Age category, years, $n(\%)$} \\
\hline$<60$ & $2(33)$ & $1(7)$ & $3(14)$ \\
\hline $60-69$ & $4(67)$ & $5(33)$ & $9(43)$ \\
\hline$\geq 70$ & 0 & $9(60)$ & $9(43)$ \\
\hline Male, $n(\%)$ & $4(67)$ & $9(60)$ & $13(62)$ \\
\hline Median longest diameter of largest lesion, $\mathrm{cm}$ (range) & $3(2-5)$ & $8(2-15)$ & $4(2-15)$ \\
\hline Median circulating lymphocytes, $10^{9} / \mathrm{L}$ (range) & $1.7(0.1-3.8)$ & $1.1(0.4-83.9)$ & $1.2(0.1-83.9)$ \\
\hline Median creatinine clearance, mL/min (range) & $108(72-140)$ & $57(36-94)$ & $70(36-140)$ \\
\hline Median number of prior therapies, $n$ (range) & $2(1-2)$ & $2(1-4)$ & $2(1-4)$ \\
\hline
\end{tabular}

TLS tumor lysis syndrome

${ }^{a}$ Nine (60\%) patients had $\geq 1$ lesion $>10 \mathrm{~cm}$ and $9(60 \%)$ patients had baseline creatinine clearance $<60 \mathrm{~mL} / \mathrm{min}$

\section{Safety}

The median treatment duration was 20.1 months (range, $<1-38$ months). There were no clinical TLS events. One 74-year-old female patient at increased risk for TLS based on 1 lesion $>10 \mathrm{~cm}$ and baseline creatinine clearance $<60 \mathrm{~mL} / \mathrm{min}$ had onset of a laboratory TLS on Day 2 of treatment. The patient had a medical history of Grade 1 hypertension, and Grade 2 atrial fibrillation, cardiac failure, chronic renal insufficiency, dyspnea, and edema of lower extremities; baseline bone marrow lymphoma involvement was $10 \%$, and baseline white blood cell count was $111.9 \times 10^{9} / \mathrm{L}$. The laboratory TLS event lasted 5 days, during which the patient was able to remain on ibrutinib $(560 \mathrm{mg})$ while holding venetoclax $(20 \mathrm{mg})$. For this patient, potassium and calcium remained within the normal range (maximum potassium $4.6 \mathrm{mmol} / \mathrm{L}$, minimum calcium $1.89 \mathrm{mmol} / \mathrm{L}$ ), while levels were outside the normal range for phosphorous (maximum $2.84 \mathrm{mmol} / \mathrm{L}$ ), uric acid (maximum $667 \mu \mathrm{mol} / \mathrm{L}$ ), and creatinine (maximum $137 \mu \mathrm{mol} / \mathrm{L}$ ) during the five-day period. On Day 7 , the patient resumed venetoclax $(20 \mathrm{mg}$ ) (Additional file 1: Figure S3); the white blood cell counts gradually decreased to $5.8 \times 10^{9} / \mathrm{L}$ on Day 22 , and ramp-up to full dose was achieved, with a best response of PR. DLTs occurred in three patients (14\%): grade 4 neutropenia lasting $>7$ days $(n=1)$, grade 4 infection $(n=1)$, and grade 3 atrial fibrillation plus grade 3 hypotension $(n=1)$.

Most AEs were low grade (grade 1/2) (Fig. 1A). Grade $\geq 3$ infections occurred in eight patients (38\%). Grade $\geq 3$ diarrhea or neutropenia occurred in seven patients each (33\%) (Fig. 1B). Grade $\geq 3$ atrial fibrillation and hemorrhage occurred in one patient each. Five patients (24\%) discontinued both study drugs due to AEs
(Additional file 1: Table S2). Four patients (19\%) discontinued treatment due to PD.

\section{Efficacy}

For all patients, ORR was 81\% (95\% CI, 58-95\%); rates were similar regardless of TLS risk (Fig. 2A). Thirteen patients achieved a CR (62\%; 95\% CI, 38-82\%); all 11 patients with detectable MRD at baseline achieved undetectable MRD. Median duration of response was 32.3 months (95\% CI, 26.5-not estimable [NE]). Median PFS was 35.0 months (95\% CI, 13.7-NE) (Fig. 2B); the 30-month PFS estimate was 60\% (95\% CI: 31-80\%). Median OS was also 35.0 months (95\% CI, 20.7-NE) (Additional file 1: Figure S4).

\section{Discussion}

Results from the SRI of the SYMPATICO study demonstrate that the combination of ibrutinib plus venetoclax is well tolerated and can be safely administered in patients with relapsed/refractory MCL, without the need for an ibrutinib lead-in. To our knowledge, this is the first time that concurrent administration of this treatment combination has been investigated. Notably, a majority of patients enrolled in the SRI cohort were in the increasedrisk category for TLS based on tumor burden. In patients with chronic lymphocytic leukemia who are in the highrisk category for TLS, three cycles of a single-agent ibrutinib lead-in have been shown to effectively debulk tumor burden prior to administration of combined ibrutinib plus venetoclax, with $94 \%$ of patients shifting from the high category at baseline to medium or low categories after lead-in [15]. In the single-arm phase 2 AIM study, which investigated the treatment of relapsed/refractory 
(A)

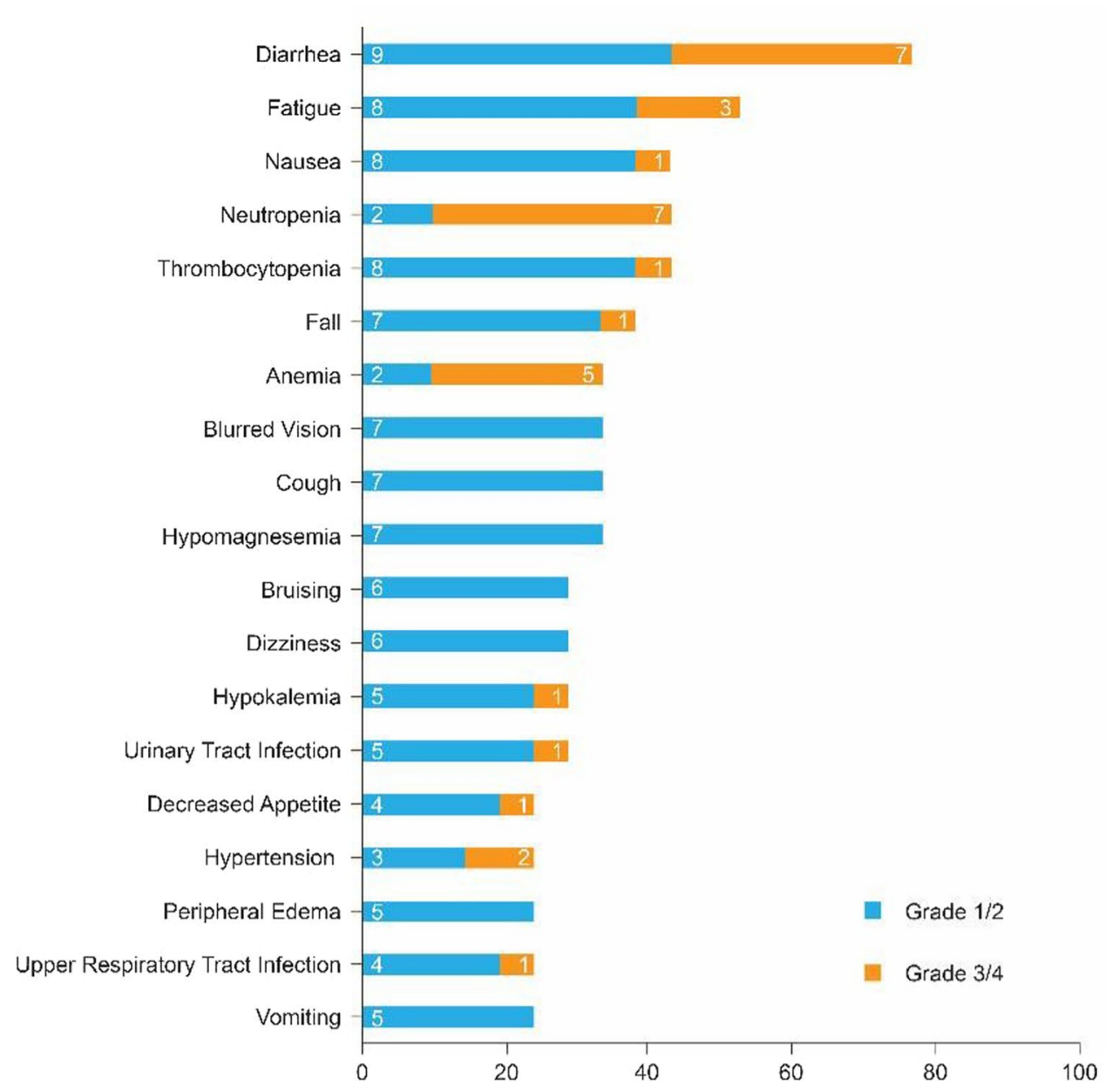

(B)

Patients, \%

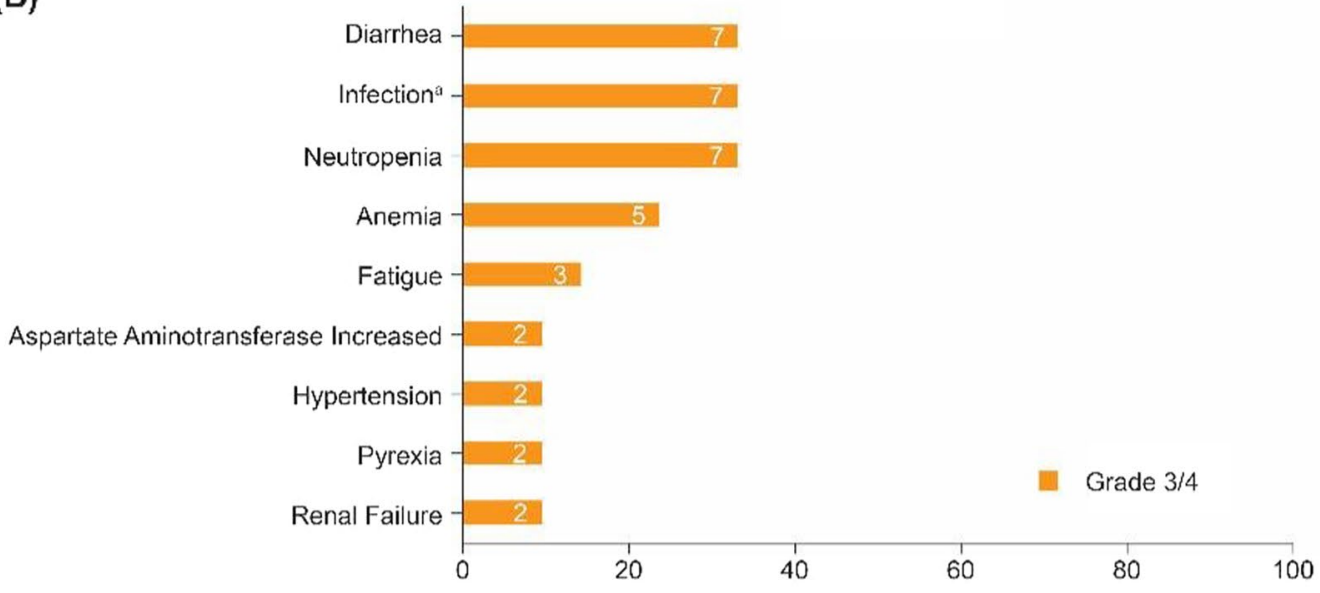

Patients, \%

Fig. 1 Most common adverse events by grade. A Any-grade treatment-emergent adverse events occurring in $>20 \%$ of all patients. B Grade 3/4

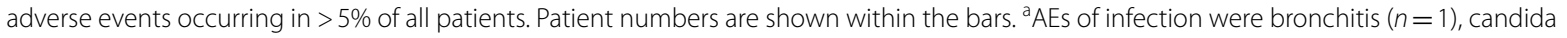
infection $(n=1)$, cellulitis $(n=1)$, fungal abscess central nervous system $(n=1$, recovered), infection (not specified, $n=1)$, pneumonia $(n=2)$, sepsis $(n=1)$, staphylococcal bacteremia $(n=1)$, upper respiratory tract infection $(n=1)$, and urinary tract infection $(n=1)$ 
(A)

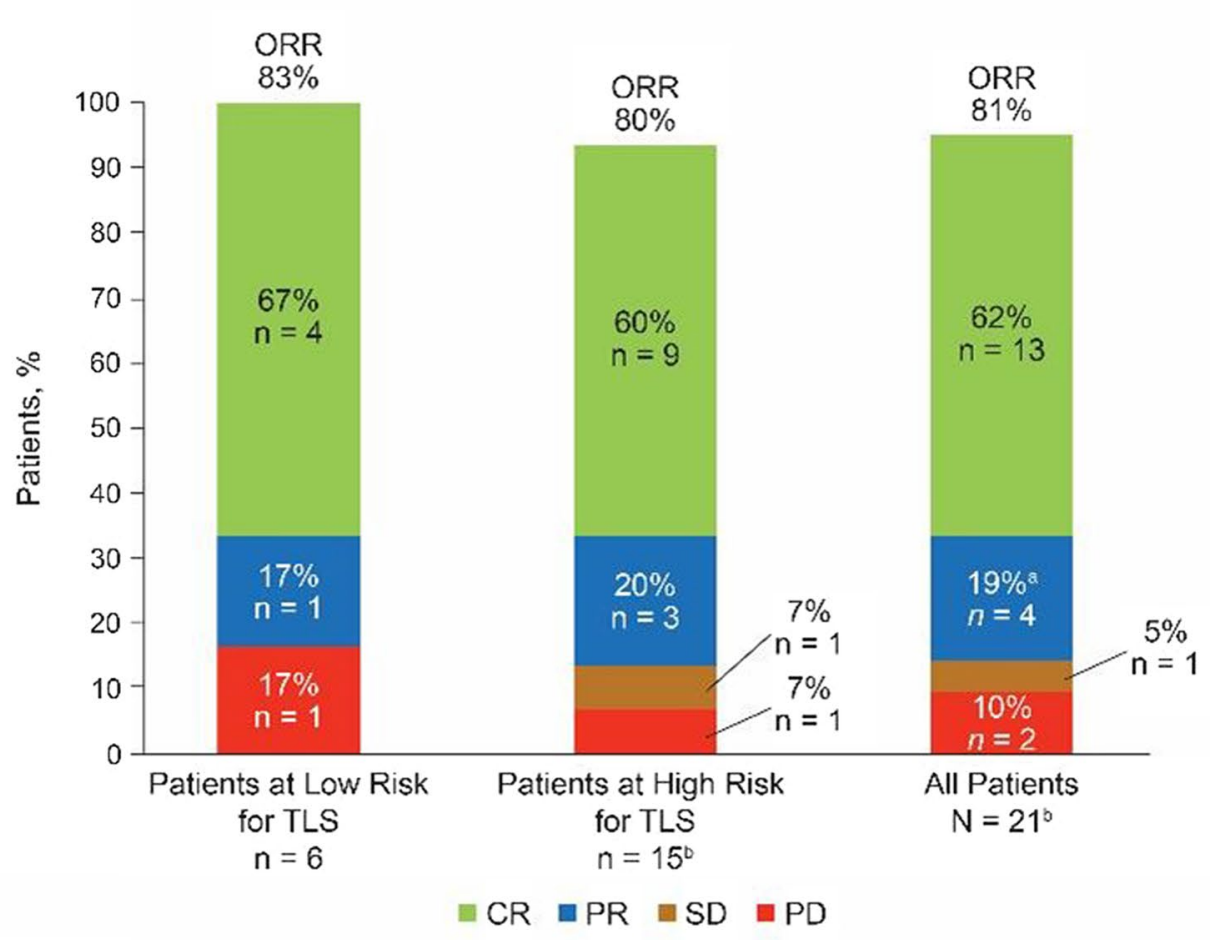

(B)

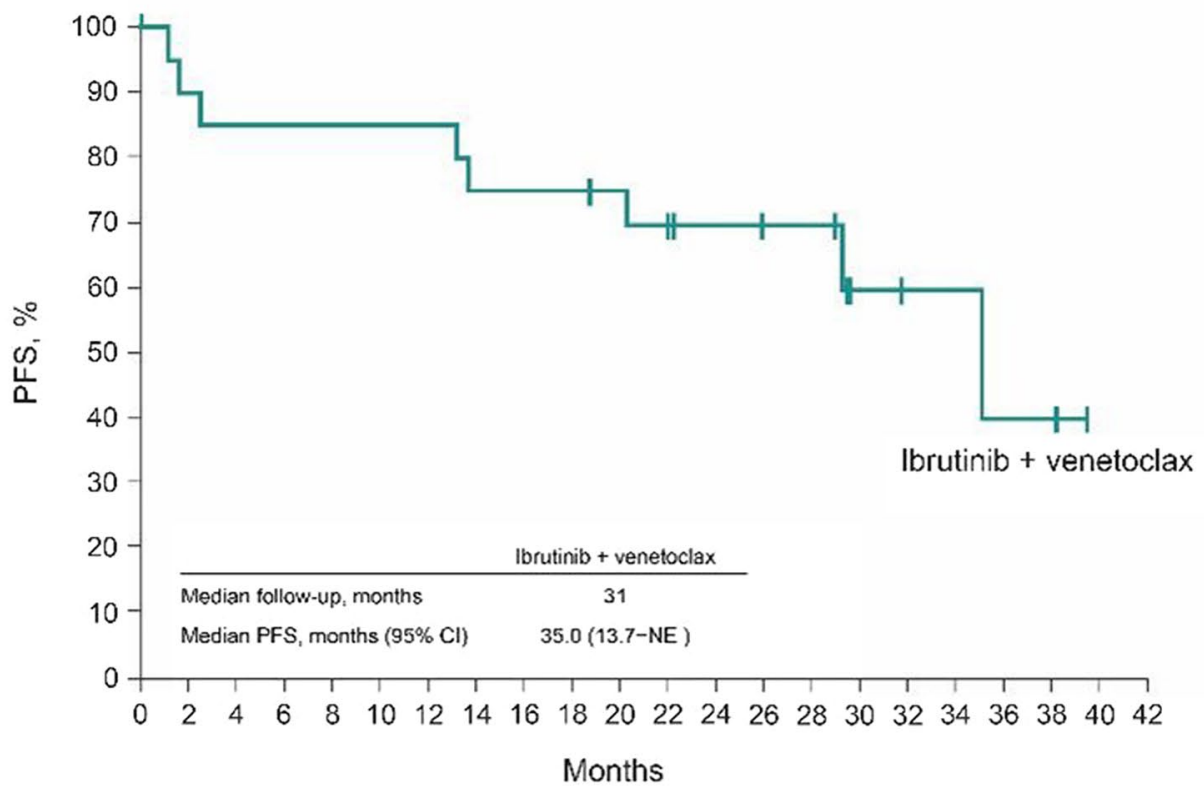

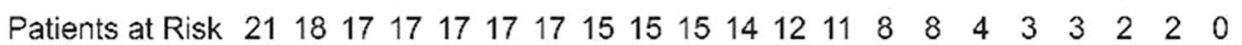

Fig. 2 Investigator-assessed efficacy outcomes. A Overall response by TLS-risk group. B PFS by Kaplan-Meier estimates. Tick marks indicate patients with censored data. Abbreviations: $\mathrm{Cl}$, confidence interval; $\mathrm{CR}$, complete response; $\mathrm{CT}$, computed tomography; $\mathrm{NE}$, not estimable; ORR, overall response rate; PD, progressive disease; PFS, progression-free survival; PR, partial response; SD, stable disease; TLS, tumor lysis syndrome. ${ }^{\mathrm{a} T w o}$ patients with a CR by CT scan are missing confirmatory bone marrow examinations and therefore are considered to have PR. ${ }^{\mathrm{b}}$ One patient was not evaluable 
MCL with ibrutinib plus venetoclax, a 4-week lead-in period with ibrutinib was utilized, and the TLS-risk category was reduced from high to low in three of seven patients [16]. However, in the same study, two patients never received venetoclax due to rapid PD during the ibrutinib lead-in period in one patient and fatal infection in the other. Given the aggressive nature of the disease, concurrent initiation of the combination is desirable.

In the current study, with the concurrent administration of ibrutinib plus venetoclax, one single instance of laboratory TLS occurred, which resolved in 5 days, and there were no cases of clinical TLS. During the SRI, DLTs were observed in three patients (neutropenia, infection, and atrial fibrillation plus hypotension); however, the DLT incidence did not exceed the prespecified threshold to necessitate an ibrutinib lead-in. Additionally, the safety profile of ibrutinib plus venetoclax was consistent with the known AEs for the individual agents, with no added toxicities. Therefore, based on these data and per study protocol algorithm, it was concluded that the double-blind randomized SYMPATICO cohort for relapsed/ refractory MCL would proceed with concurrent initiation of ibrutinib and venetoclax treatment.

Notably, efficacy outcomes in the SYMPATICO SRI were similar to those in the phase 2 AIM study, despite having shorter follow-up ( 31 vs. 37.5 months) and without an ibrutinib lead-in. In the current study, the CR rate was $62 \%$, and AIM reported a CR rate of $71 \%$, while the median PFS was slightly longer in the SYMPATICO SRI cohort versus AIM (35 vs. 29 months, respectively) [17]. This novel combination appears to be more effective than previously reported single-agent treatment with either drug in relapsed/refractory MCL, as patients treated with single-agent ibrutinib reached CR rates of $19-28 \%$ [3-5] and a median PFS of 12.5-15.6 months $[4,5]$ whereas venetoclax monotherapy induced CR rates of $21 \%$ and a median PFS of 14 months [7]. In addition, these preliminary efficacy data from the SRI cohort represent an advancement in either CR rate and/or sustained PFS compared to recent treatment options for patients with relapsed/refractory $\mathrm{MCL}$, including the BTK inhibitors zanubrutinib (68.6\% CR rate; 22.1 months median PFS) [18] and acalabrutinib (43\% CR rate; 20 months median PFS) [19] or KTE-X19 (anti-CD19 chimeric antigen receptor T-cell therapy; $67 \% \mathrm{CR}$ rate; median PFS not reached at 12.3 months of follow-up) [20].

In conclusion, SYMPATICO SRI results demonstrate that ibrutinib plus venetoclax was well tolerated without an ibrutinib lead-in. This combination represents a once-daily, all-oral chemotherapy-free regimen for patients with relapsed/refractory MCL. The randomized portion of the SYMPATICO study is evaluating ibrutinib plus venetoclax versus ibrutinib plus placebo in patients with relapsed/refractory MCL. A single-arm, openlabel cohort in previously untreated patients with MCL, including those with TP53 mutations, is also ongoing.

\section{Abbreviations}

AE: adverse event; BTK: Bruton's tyrosine kinase; Cl: confidence interval; CR: complete response; CT: computed tomography; DLT: dose-limiting toxicity; ECOG: Eastern Cooperative Oncology Group; HR: hazard ratio; MCL: mantle cell lymphoma; MRD: minimal residual disease; NE: not estimable; ORR: overall response rate; OS: overall survival; PD: progressive disease; PET: positron emission tomography; PFS: progression-free survival; R/R: relapsed/refractory; SRI: safety run-in; TLS: tumor lysis syndrome.

\section{Supplementary Information}

The online version contains supplementary material available at https://doi. org/10.1186/s13045-021-01188-x.

Additional file 1. SUPPLEMENTARY INFORMATION: Supplemental Methods; Table S1. Patient Disposition; Table S2. Safety Summary; Figure S1. SYMPATICO study schemas; Figure S2. Schema to determine randomized phase 3 dosing; Figure $\mathbf{S 3}$. Metabolic laboratory values and abnormalities in one patient with laboratory TLS; Supplementary Figure S4. Overall survival.

\section{Acknowledgements}

We thank the patients who participated in the study and their supportive families, as well as the investigators and clinical research staff from the study centers. Editorial support was provided by Emily Chastain, Ph.D., an employee of Pharmacyclics LLC, an AbbVie Company.

\section{Authors' contributions}

MW and CST designed the study in collaboration with the study sponsor; $R R, R C, L K, W J, K L W, M B, G P C, P E$, and FP contributed to data collection; YL performed the data analyses; $Y L, K E$, and JKN confirmed the accuracy of the data and compiled it for analysis; all authors had access to the data and were involved in the interpretation of data, contributed to the manuscript review and revisions, and approved the final version for submission. All authors read and approved the final manuscript.

Funding

This study was sponsored by Pharmacyclics LLC, an AbbVie Company. The study sponsor in collaboration with the authors designed the study, performed data collection, analysis, interpretation, and review of the manuscript.

\section{Availability of data and materials}

Requests for access to individual participant data from clinical studies conducted by Pharmacyclics LLC, an AbbVie Company, can be submitted through Yale Open Data Access Project site (http://yoda.yale.edu).

\section{Declarations}

\section{Ethics approval and consent to participate}

The study was conducted according to the principles of the Declaration of Helsinki and the Good Clinical Practice guidelines from the International Conference on Harmonization. The protocol was approved by institutional review boards or independent ethics committees at participating institutions, and all patients provided written informed consent.

\section{Consent for publication}

Not applicable.

\section{Competing interests}

MW: honoraria from Janssen, AstraZeneca, OMI, Targeted Oncology, OncLive, Dava Oncology, Beijing Medical Award Foundation, Lu Daopei Medical Group, and Pharmacyclics LLC, an AbbVie Company; consulting or advisory role 
with Celgene, Janssen, AstraZeneca, MORE Health, Pulse Biosciences, Nobel Insights, Guidepoint Global, Kite Pharma, Juno Therapeutics, Loxo Oncology, InnoCare, Oncternal, and Pharmacyclics LLC, an AbbVie Company; research funding from Janssen, AstraZeneca, Acerta Pharma, Kite Pharma, Juno Therapeutics, Celgene, Loxo Oncology, VelosBio, Verastem, Molecular Templates, Biolnvent, Oncternal, and Pharmacyclics LLC, an AbbVie Company; and travel accommodations from Janssen, Celgene, Juno Therapeutics, Kite Pharma, Loxo Oncology, VelosBio, Verastem, Molecular Templates, Biolnvent, Oncternal, AstraZeneca, Acerta Pharma, and Pharmacyclics LLC, an AbbVie Company. RR: consulting or advisory role with Pharmacyclics LLC, an AbbVie Company; and research funding from Janssen and Pharmacyclics LLC, an AbbVie Company. RC: nothing to disclose. LK: employment with Aguettant; honoraria from Amgen, AbbVie, Celgene, Janssen, Takeda, and Sanofi; consulting or advisory role with Amgen, Celgene, Janssen, and Takeda; and travel accommodations from Amgen, Janssen, and Takeda. GC: consulting or advisory role with Bristol Myers Squibb; research funding from Merck Serono, Bristol Myers Squibb, Hutchison MediPharma, Regeneron, Isofol, AstraZeneca, Servier, and Pharmacyclics LLC, an AbbVie Company. WJ: consulting or advisory role with Sandoz Novartis, BeiGene, Janssen, Acerta, AstraZeneca, Loxo, and Epizyme; research funding from AbbVie, Acerta, Bayer, BeiGene, Janssen, MEl Pharma, Takeda, Telios, TG Therapeutics, and Pharmacyclics LLC, an AbbVie Company. KLW: consulting or advisory role with Amgen, Janssen, and AbbVie. MB: honoraria from Teva Pharma, Celltrion, and Roche Pharmaceuticals; consulting or advisory role with Celltrion, and Roche Pharmaceuticals; travel accommodations from Celltrion, Roche Pharmaceuticals, Takeda, and Gilead. GPC: honoraria from Roche, Takeda, Gilead, Pfizer, Bristol Myers Squibb, Merck Sharp \& Dohme, Celleron, ADC Therapeutics, and Novartis; consulting or advisory role with Roche, Takeda, Gilead, Bristol Myers Squibb, Merck Sharp \& Dohme, Celleron, and ADC Therapeutics; research funding from Bristol Myers Squibb, Celleron, Merck Sharp \& Dohme, Celgene, and Amgen; speakers bureau with Roche, Takeda, Bristol Myers Squibb, Novartis, and Gilead; travel accommodations from Roche and Takeda. PE: nothing to disclose. FP: nothing to disclose. YL, KE, and JKN: employment with Pharmacyclics LLC, an AbbVie Company; and stock ownership in AbbVie. CST: honoraria from AbbVie, Janssen, Loxo, and BeiGene; consulting or advisory role with Janssen, Loxo, Roche, BeiGene, and AbbVie; and research funding from BeiGene, Janssen, and AbbVie.

\section{Author details}

${ }^{1}$ Department of Lymphoma and Myeloma, University of Texas MD Anderson Cancer Center, 1515 Holcombe Blvd \#368, Houston, TX 77030, USA. ${ }^{2}$ University of Tennessee, Knoxville, TN, USA. ${ }^{3}$ City of Hope National Medical Center, Duarte, CA, USA. ${ }^{4}$ Centre Hospitalier Lyon Sud, Lyon, France. ${ }^{5}$ Olivia NewtonJohn Cancer Centre, Heidelberg, VIC, Australia. ${ }^{6}$ Sklodowska Curie National Research Institute of Oncology, Kraków, Poland. 'Z Ziekenhuis Netwerk Antwerpen, Antwerp, Belgium. ${ }^{8}$ Nottinghamshire University Hospitals, Nottingham, UK. ${ }^{9} \mathrm{NIHR}$ Oxford Biomedical Research Centre, Churchill Hospital, Oxford, UK. ${ }^{10}$ Icon Cancer Centre, South Brisbane, QLD, Australia. ${ }^{11}$ Centre Antoine Lacassagne, Nice Cedex 2, France. ${ }^{12}$ Pharmacyclics LLC, an AbbVie Company, Sunnyvale, CA, USA. ${ }^{13}$ Peter MacCallum Cancer Centre, Royal Melbourne Hospital, St Vincent's Hospital, University of Melbourne, Melbourne, VIC, Australia.

Received: 27 August 2021 Accepted: 12 October 2021

Published online: 30 October 2021

\section{References}

1. IMBRUVICA (ibrutinib) [prescribing information]. Sunnyvale: Pharmacyclics LLC; 2021.

2. Wang ML, Rule S, Martin P, Goy A, Auer R, Kahl BS, Jurczak W, Advani RH, Romaguera JE, Williams ME, Barrientos JC, Chmielowska E, Radford J, Stilgenbauer S, Dreyling M, Jedrzejczak WW, Johnson P, Spurgeon SE, Li L, Zhang L, Newberry K, Ou Z, Cheng N, Fang B, McGreivy J, Clow F, Buggy JJ, Chang BY, Beaupre DM, Kunkel LA, Blum KA. Targeting BTK with ibrutinib in relapsed or refractory mantle-cell lymphoma. N Engl I Med. 2013;369(6):507-16. https://doi.org/10.1056/NEJMoa1306220.

3. Dreyling M, Jurczak W, Jerkeman M, Silva RS, Rusconi C, Trneny M, Offner F, Caballero D, Joao C, Witzens-Harig M, Hess G, Bence-Bruckler I, Cho S-G, Bothos J, Goldberg JD, Enny C, Traina S, Balasubramanian S, Bandyopadhyay N, Sun S, Vermeulen J, Rizo A, Rule S. Ibrutinib versus temsirolimus in patients with relapsed or refractory mantle-cell lymphoma: an international, randomised, open-label, phase 3 study. Lancet. 2016;387(10020):770-8. https://doi.org/10.1016/S0140-6736(15)00667-4.

4. Rule S, Jurczak W, Jerkeman M, Rusconi C, Trneny M, Offner F, Caballero D, Joao C, Witzens-Harig M, Hess G, Bence-Bruckler I, Cho S-G, Thieblemont C, Zhou W, Henninger T, Goldberg J, Vermeulen J, Dreyling M. Ibrutinib versus temsirolimus: 3 -year follow-up of patients with previously treated mantle cell lymphoma from the phase 3 , international, randomized, open-label RAY study. Leukemia. 2018;32(8):1799-803. https://doi.org/10. 1038/s41375-018-0023-2.

5. Rule S, Dreyling MH, Goy A, Hess G, Auer R, Kahl BS, Hernandez-Rivas JA, Qi K, Deshpande S, Parisi L, Wang ML. Long-term outcomes with ibrutinib versus the prior regimen: a pooled analysis in relapsed/refractory $(R / R)$ mantle cell lymphoma (MCL) with up to 7.5 years of extended follow-up. Blood. 2019;134(Supplement_1):1538. https://doi.org/10.1182/ blood-2019-124691.

6. VENCLEXTA (venetoclax) [prescribing information]. North Chicago, LL: AbbVie Inc; 2019.

7. Davids MS, Roberts AW, Seymour JF, Pagel JM, Kahl BS, Wierda WG, Puvvada S, Kipps TJ, Anderson MA, Salem AH, Dunbar M, Zhu M, Peale F, Ross JA, Gressick L, Desai M, Kim SY, Verdugo M, Humerickhouse RA, Gordon GB, Gerecitano JF. Phase I first-in-human study of venetoclax in patients with relapsed or refractory non-Hodgkin lymphoma. J Clin Oncol. 2017;35(8):826-33. https://doi.org/10.1200/JCO.2016.70.4320.

8. Portell CA, Axelrod M, Brett LK, Gordon VL, Capaldo B, Xing JC, Bekiranov S, Williams ME, Weber MJ. Synergistic cytotoxicity of ibrutinib and the BCL2 antagonist, ABT-199(GDC-0199) in mantle cell lymphoma (MCL) and chronic lymphocytic leukemia (CLL): molecular analysis reveals mechanisms of target interactions. Blood. 2014;124(21):509. https://doi. org/10.1182/blood.V124.21.509.509.

9. Roberts AW, Davids MS, Pagel JM, Kahl BS, Puvvada SD, Gerecitano JF, Kipps TJ, Anderson MA, Brown JR, Gressick L, Wong S, Dunbar M, Zhu M, Desai MB, Cerri E, Heitner Enschede S, Humerickhouse RA, Wierda WG, Seymour JF. Targeting BCL2 with venetoclax in relapsed chronic lymphocytic leukemia. N Engl J Med. 2016;374(4):311-22. https://doi.org/ 10.1056/NEJMoa1513257.

10. Davids MS, Hallek M, Wierda W, Roberts AW, Stilgenbauer S, Jones JA, Gerecitano JF, Kim SY, Potluri J, Busman T, Best A, Verdugo ME, Cerri E, Desai M, Hillmen P, Seymour JF. Comprehensive safety analysis of venetoclax monotherapy for patients with relapsed/refractory chronic lymphocytic leukemia. Clin Cancer Res. 2018;24(18):4371-9. https://doi.org/10.1158/ 1078-0432.CCR-17-3761.

11. Wierda WG, Byrd JC, O'Brien S, Coutre S, Barr PM, Furman RR, Kipps TJ, Burger JA, Stevens DA, Sharman J, Ghia P, Flinn IW, Zhou C, Ninomoto J, James DF, Tam CS. Tumour debulking and reduction in predicted risk of tumour lysis syndrome with single-agent ibrutinib in patients with chronic lymphocytic leukaemia. Br J Haematol. 2019;186(1):184-8. https://doi.org/10.1111/bjh.15791.

12. Grajales-Cruz AF, Chavez J, Volpe VO, Sandoval-Sus J, Shah B, Bello C, Sokol L, Fisher S, Nair RR, Seongseok Y, Saeed H, Isenalumhe L, Turba EP, Nodzon L, Ibarz JP. Venetoclax in relapsed/refractory chronic lymphocytic leukemia: a retrospective, single-institution analysis based on risk features and debulking strategy with anti-CD20 monoclonal antibody. Blood. 2019:134(1):5484. https://doi.org/10.1182/blood-2019-129260.

13. Howard SC, Jones DP, Pui CH. The tumor lysis syndrome. N Engl J Med. 2011;364(19):1844-54. https://doi.org/10.1056/NEJMra0904569.

14. Cheson BD, Fisher RI, Barrington SF, Cavalli F, Schwartz LH, Zucca E, Lister TA, Alliance, Australasian Leukaemia and Lymphoma Group, Eastern Cooperative Oncology Group, European Mantle Cell Lymphoma Consortium, Italian Lymphoma Foundation, European Organisation for Research, Treatment of Cancer/Dutch Hemato-Oncology Group, Grupo Español de Médula Ósea, German High-Grade Lymphoma Study Group, German Hodgkin's Study Group, Japanese Lymphorra Study Group, Lymphoma Study Association, NCIC Clinical Trials Group, Nordic Lymphoma Study Group, Southwest Oncology Group, United Kingdom National Cancer Research Institute. Recommendations for initial evaluation, staging, and response assessment of Hodgkin and non-Hodgkin lymphoma: the Lugano classification. J Clin Oncol. 2014;32(27):3059-68. https://doi.org/ 10.1200/JCO.2013.54.8800.

15. Ghia P, Allan JN, Siddiqi T, Kipps TJ, Jacobs R, Opat S, Barr PM, Tedeschi A, Trentin L, Bannerji R, Jackson S, Kuss B, Moreno C, Szafer-Glusman E, Russell K, Zhou C, Suzuki Ninomoto J, Dean JP, Wierda WG, Tam CSL. 
Fixed-duration (FD) first-line treatment (tx) with ibrutinib (I) plus venetoclax (V) for chronic lymphocytic leukemia (CLL)/small lymphocytic lymphoma (SLL): primary analysis of the FD cohort of the phase 2 captivate study. J Clin Oncol. 2021;39(15_suppl):7501. https://doi.org/10.1200/JCO. 2021.39.15_suppl.7501.

16. Tam CS, Anderson MA, Pott C, Agarwal R, Handunnetti S, Hicks RJ, Burbury K, Turner G, Di lulio J, Bressel M, Westerman D, Lade S, Dreyling M, Dawson S-J, Dawson MA, Seymour J, Roberts AW. Ibrutinib plus venetoclax for the treatment of mantle-cell lymphoma. N Engl J Med. 2018;378(13):1211-23. https://doi.org/10.1056/NEJMoa1715519.

17. Handunnetti SM, Anderson MA, Burbury K, Hicks RJ, Birbirsa B, Bressel M, Di Iulio J, Alan Westerman DA, Lade S, Agarwal R, Dawson MA, Dawson S-J, Koldej R, Ritchie D, Seymour JF, Roberts AW, Tam CS. Three year update of the phase II ABT-199 (venetoclax) and ibrutinib in mantle cell lymphoma (AIM) study. Blood. 2019;134(Supplement_1):756. https://doi. org/10.1182/blood-2019-126619.

18. Song Y, Zhou K, Zou D, Zhou J, Hu J, Yang H, Zhang H, Ji J, Xu W, Jin J, Lv F, Feng R, Gao S, Guo H, Zhou L, Elstrom R, Huang J, Novotny W, Wei R, Zhu J.Treatment of patients with relapsed or refractory mantle-cell lymphoma with zanubrutinib, a selective inhibitor of Bruton's tyrosine kinase.
Clin Cancer Res. 2020;26(16):4216-24. https://doi.org/10.1158/1078-0432. cCr-19-3703.

19. Wang M, Rule S, Zinzani PL, Goy A, Casasnovas O, Smith SD, Damaj G, Doorduijn JK, Lamy T, Morschhauser F, Panizo C, Shah B, Davies A, Eek R, Dupuis J, Jacobsen E, Kater AP, Le Gouill S, Oberic L, RobakT, Jain P, Frigault MF, Izumi R, Nguyen D, Patel P, Yin M, Długosz-Danecka M. Durable response with single-agent acalabrutinib in patients with relapsed or refractory mantle cell lymphoma. Leukemia. 2019;33(11):2762-6. https:// doi.org/10.1038/s41375-019-0575-9.

20. Wang M, Munoz J, Goy A, Locke FL, Jacobson CA, Hill BT, Timmerman JM, Holmes H, Jaglowski S, Flinn IW, McSweeney PA, Miklos DB, Pagel JM, Kersten M-J, Milpied N, Fung H, Topp MS, Houot R, Beitinjaneh A, Peng W, Zheng L, Rossi JM, Jain RK, Rao AV, Reagan PM. KTE-X19 CART-cell therapy in relapsed or refractory mantle-cell lymphoma. N Engl J Med. 2020;382(14):1331-42. https://doi.org/10.1056/NEJMoa1914347.

\section{Publisher's Note}

Springer Nature remains neutral with regard to jurisdictional claims in published maps and institutional affiliations.
Ready to submit your research? Choose BMC and benefit from:

- fast, convenient online submission

- thorough peer review by experienced researchers in your field

- rapid publication on acceptance

- support for research data, including large and complex data types

- gold Open Access which fosters wider collaboration and increased citations

- maximum visibility for your research: over $100 \mathrm{M}$ website views per year

At BMC, research is always in progress.

Learn more biomedcentral.com/submissions 